\title{
High-Resolution Terahertz Spectroscopy of Water Vapor with Different Humidity Levels under Normal Atmospheric Conditions and in a Vapor-Liquid Coexistence at Reduced Pressure
}

\author{
Tadao Tanabe*, Hiromu Matsumoto, Shintaro Yudate and Yutaka Oyama \\ Department of Materials Science, Tohoku University, Japan
}

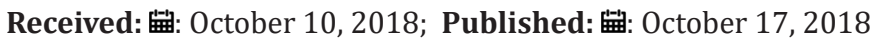

*Corresponding author: Tadao Tanabe, Department of Materials Science, Graduate School of Engineering, Tohoku University, AramakiAza Aoba 6-6-11-1021, Sendai 980-8579, Japan

\begin{abstract}
We have developed a continuous-wave (CW) terahertz (THz) radiation source that is excited using a laser diode (LD). With this source, the radiation is generated via difference frequency generation (DFG) under non-collinear phase matching in gallium phosphide (GaP). A distributed feedback (DFB) LD and an external cavity laser diode (ECLD) were used as the excitation light sources, and these, together with a polarization maintaining (PM) ytterbium-doped fiber amplifier, had linewidths of less than $4 \mathrm{MHz}$. The narrow linewidth means that the THz radiation can be used for high resolution spectroscopy. As an example of this, the spectrum for a mixture of saturated water vapor and ethanol at room temperature was obtained, where the spectral absorption lines for water and ethanol at around $2.46 \mathrm{THz}$ could be distinguished. Furthermore, the THz spectra of water vapor at different humidity levels were measured under normal atmospheric conditions. From these we obtained the dependences on humidity of the position of the absorption peak, its FWHM and the intensity of the water vapor absorption band due to the rotational transition of $4_{32} \leftarrow 4_{23}$. We also show the results for time-dependent THz spectra of water vapor in a vapor-liquid coexistence in a chamber at reduced pressure, where an absorption peak, probably due to an intermediate state of the water molecules, appeared around $2.585 \mathrm{THz}$ just after reducing the pressure in the chamber.
\end{abstract}

Keywords: Continuous-Wave Terahertz; Spectroscopy; Water Vapor; Humidity; Vapor-Liquid Coexistence

Abbreviations: GaP: Gallium Phosphide; CW: Continuous-Wave; THz: Terahertz; DFG: Difference Frequency Generation; DFB: Distributed Feedback; ECLD: External Cavity Laser Diode; NLO: Nonlinear Optical; QCL: Quantum Cascade Lasers; LD: Laser Diode

\section{Introduction}

The terahertz $(\mathrm{THz})$ region of the electromagnetic spectrum is distributed from 0.1 to $10 \mathrm{THz}$. It has high transmittance in nonmetallic materials, and the frequency of $\mathrm{THz}$ radiation corresponds to the energy of weak intermolecular bonding and to transitions between rotational energy levels. Because of these characteristics, it is expected that $\mathrm{THz}$ radiation can be applied to specialized spectroscopic imaging [1], high speed telecommunications [2] and non-destructive inspection and measurement [3-5]. To the best of our knowledge, THz radiation presents no serious danger for the human body. Furthermore, there are many interesting applications for $\mathrm{THz}$ spectroscopy, for example, unambiguous monitoring of toxic materials without ionization, the detection and analysis of smoke in the atmosphere, and detecting small amounts of hazardous impurities [6-10]. The spectral lines for the rotational transitions of gas molecules are sharp and dense, so high resolution spectroscopy is necessary. Many THz radiation sources have been developed based on nonlinear optical (NLO) processes such as difference frequency generation (DFG) [11-12]. Furthermore, quantum cascade lasers (QCL) have been realized [13-15].

The advantages of DFG are that it operates at room-temperature, the radiation is monochromatic and the frequency is tunable over a wide range. In particular, the $\mathrm{THz}$ radiation from GaP pumped by a CW (continuous-wave) laser diode (LD) has very narrow linewidths that enable high-resolution $\mathrm{THz}$ spectra to be done $[16,17]$. To develop practical applications that use THz spectroscopy under normal atmospheric conditions, clear THz spectra of the omnipresent water vapor should be obtained. The absorption lines of water vapor are known to broaden with increasing pressure, so many of the previous theoretical and experimental studies reporting on the THz spectra of water vapor were done under ideal conditions, that is, at low pressure [7,18-22]. In this study, we used the system mentioned above, GaP pumped by a CW LD, as a THz source to make spectral measurements on a mixture of saturated 
water vapor and ethanol at room temperature. We also measured the $\mathrm{THz}$ spectra of water vapor at different humidity levels at atmospheric pressure and in a vapor-liquid coexistence at reduced pressure.

\section{Experimental}

Figure 1 shows the optical setup for the $\mathrm{THz}$ spectral measurement system. The pump and signal lasers are an ECLD (external cavity laser diode) and a DFB (distribution feedback) laser, respectively. The wavelength range of the DFB laser was automatically tuned from 1058 to $1061 \mathrm{~nm}$ (0.6 THz) with a linewidth of $3 \mathrm{MHz}$ by changing the temperature of the laser. The wavelength of the ECLD was manually tuned from 1020 to $1080 \mathrm{~nm}$ with a linewidth of $4 \mathrm{MHz}$. Two polarization maintaining ytterbium doped fiber amplifiers (FAs) were used and each laser diode was amplified up to $2 \mathrm{~W}$. The $\mathrm{THz}$ frequency was changed continuously by tuning the wavelength of the DFB laser by temperature control. The details are described in our previous paper [12,17]. The GaP crystal was cut into a rectangle measuring $10 \mathrm{~mm}$ in the $<110>$ direction and $3 \mathrm{~mm}$ in the $<001>$ direction. A polarizing beam splitter was used to combine the pump beam with the signal beam. The incident beam angle was aligned roughly parallel to the $<110>$ direction and the two lasers were focused onto the GaP surface at a phase matching angle. The $\mathrm{CW} \mathrm{THz}$ radiation generated was reflected by parabolic mirrors, and the THz signal was detected using a liquid-helium cooled $\mathrm{Si}$ bolometer.

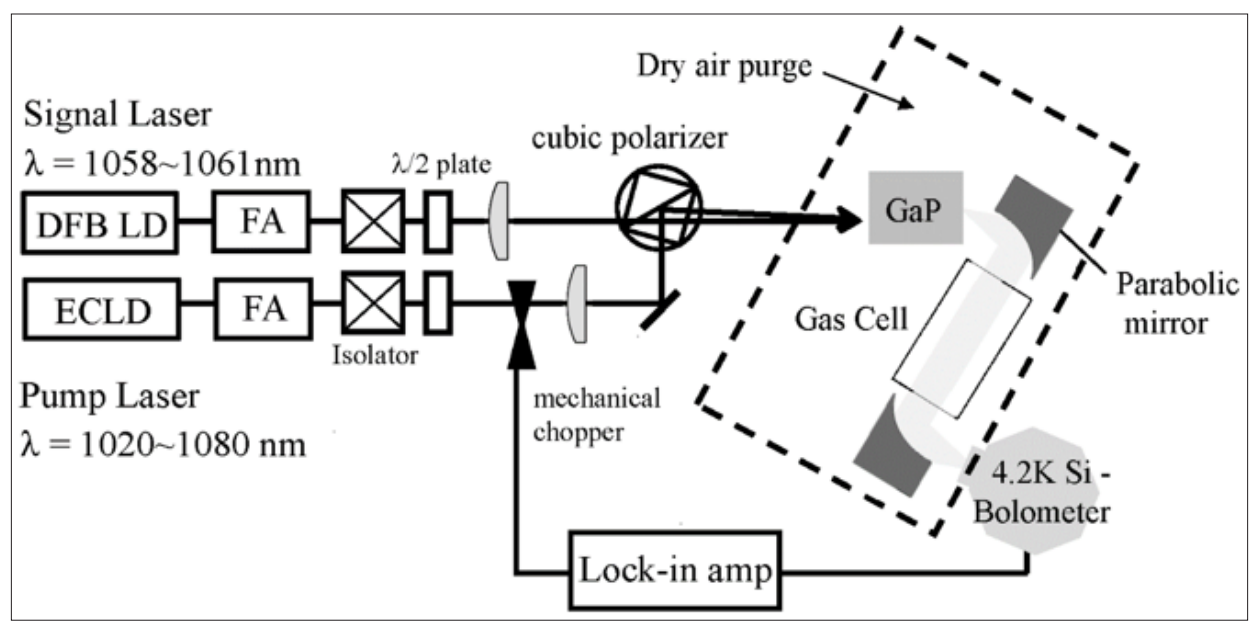

Figure 1: The optical setup for the THz spectroscopy measurement system for water vapor using LD pumped CW THz radiation.

A gas cell with $4 \mathrm{~mm}$ thick polyethylene windows containing the ethanol/water vapor mixture was placed between the two parabolic mirrors in order to measure the spectra. The optical path length and volume of the cell were $232 \mathrm{~mm}$ and $0.12 \mathrm{~L}$, respectively. $\mathrm{THz}$ spectra of the water vapor were taken at different humidity levels at atmospheric pressure, with an optical path length of $530 \mathrm{~mm}$. The humidity was controlled by changing the flow rate of dry air. The absolute humidity of dry air with a dew point of $\leq-70$ ${ }^{\circ} \mathrm{C}$ is $4.74 \times 10^{-3} \mathrm{~g} / \mathrm{m}^{3}$, which is $0.019 \%$ relative humidity at $20^{\circ} \mathrm{C}$. The humidity was measured using a thin film capacitive relative humidity sensor. The pressure in the gas cell was reduced by using a diaphragm pump, and the pressure was measured by a bourdon tube pressure gage. Before evacuation, liquid water was introduced into the cell. Spectra of the water vapor were measured before and after evacuation, where the pressure in the gas cell under vaporliquid equilibrium conditions at room temperature was $14.6 \mathrm{kPa}$.

\section{Results and Discussion}

Figure 2(a) shows the THz spectrum of saturated water vapor and ethanol at atmospheric pressure and room temperature in the frequency range from 2.4 to $2.68 \mathrm{THz}$ in $336 \mathrm{MHz}$ steps. Several absorption lines are observed in this frequency region. The peaks shown by closed circles ( $)$ are due to the rotational energy state transitions of water. The peaks shown by solid triangles $(\boldsymbol{\Delta})$ are attributed to the rotational energy state transition lines of ethanol gas. The absorption spectrum around $2.46 \mathrm{THz}$ was measured with a fine frequency step of $87 \mathrm{MHz}$, as shown in Figure 2(b). The absorption bands were separated with a Lorentz function, revealing peaks at $2.4658 \mathrm{THz}$ and $2.4682 \mathrm{THz}$. This result suggests that the spectral resolution is within $2.4 \mathrm{GHz}$. Figure 3 shows the THz spectra of water vapor with different humidities at atmospheric pressure. The spectra were measured in the frequency range from 2.41 to $2.53 \mathrm{THz}$ as a function of humidity at room temperature. As shown in Figures $3 \mathrm{a} \& 3 \mathrm{~b}$, at humidities of 3.69 and $1.55 \mathrm{~g} / \mathrm{m}^{3}$, absorption peaks appear at around $2.40 \mathrm{THz}, 2.44 \mathrm{THz}, 2.45 \mathrm{THz}$ and $2.47 \mathrm{THz}$. These absorption bands are assigned to the following rotational transitions of water; $\left(4_{04} \leftarrow 3_{13}\right),\left(9_{36} \leftarrow 9_{27}\right),\left(8_{44} \leftarrow 8_{35}\right)$ and $\left(4_{32} \leftarrow 3_{23}\right)$, respectively [23].

These absorption peaks are hardly observed at humidities less than $0.16 \mathrm{~g} / \mathrm{m}^{3}$. The position of the $2.47 \mathrm{THz}$ absorption peak shifts to lower values and the FWHM becomes smaller as the number of surrounding water molecules decreases, and the nitrogen and oxygen start to have an effect on the interactions between the water molecules. Figure 3(e) shows the dependences of the peak position, the FWHM and the intensity of the absorption band on humidity. The absorbance and peak frequency are proportional to 
the humidity of water vapor below $0.5 \mathrm{~g} / \mathrm{m}^{3}$. This indicates that the THz absorption is based on the Beer-Lambert law. For humidities above $1.5 \mathrm{~g} / \mathrm{m}^{3}$, the $\mathrm{THz}$ absorption is saturated due to interactions between the water molecules, and the FWHM is broadened by this increase in molecular interactions. The interaction is due to the polarity of the water molecules, and this is larger than that of alcohol. The interactions for alcohol molecules, which have more carbon atoms with a small proportion of $-\mathrm{OH}$, is weaker. Figure 4 shows the THz spectra of water in a vapor-liquid coexistence at a pressure of $14.6 \mathrm{kPa}$.
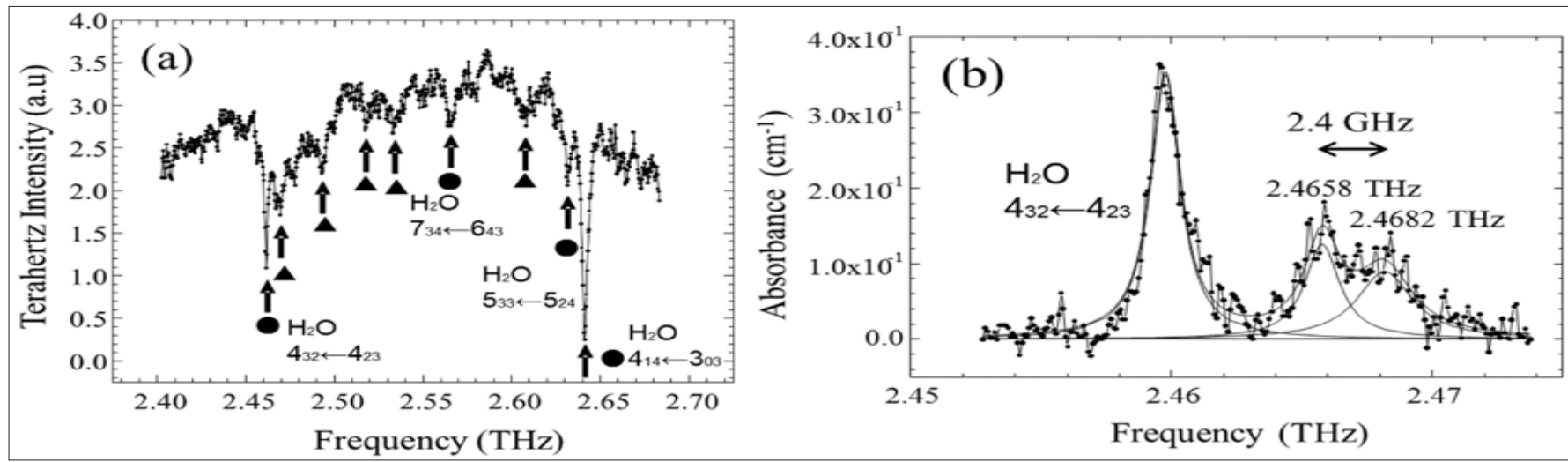

Figure 2: $\mathrm{THz}$ spectra of a mixture of saturated water vapor and ethanol at atmospheric pressure with frequency steps of $3.36 \times 10^{-4} \mathrm{THz}(\mathrm{a})$ and $8.7 \times 10^{-5} \mathrm{THz}(\mathrm{b})$, respectively.

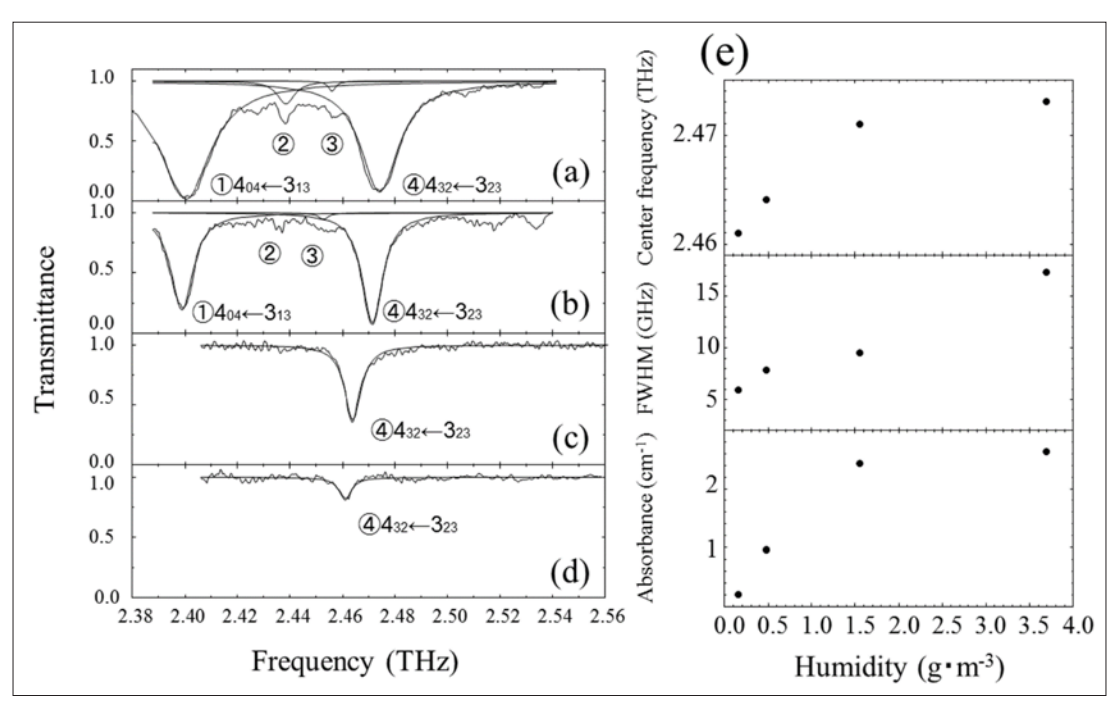

Figure 3: THz spectra of water vapor at different humidity levels at atmospheric pressure. The humidity is $3.69 \mathrm{~g} / \mathrm{m}^{3}(\mathrm{a}), 1.55 \mathrm{~g} / \mathrm{m}^{3}(\mathrm{~b}), 0.480$ $\mathrm{g} / \mathrm{m}^{3}$ (c) and $0.160 \mathrm{~g} / \mathrm{m}^{3}(\mathrm{~d})$, respectively. (e) Dependences on humidity of the absorption peak position, the FWHM, and the intensity of the absorption band around $2.47 \mathrm{THz}$ due to the rotational transition of $4_{32} \leftarrow 4_{23}$.

The $\mathrm{THz}$ spectrum at atmospheric pressure is shown in Figure 4(a). An absorption peak, with a full width at half maximum (FWHM) of $5.92 \mathrm{GHz}$, is located at $2.569 \mathrm{THz}$. This is assigned to the rotational transition of water $\left(7_{34} \leftarrow 6_{43}\right)$ [23]. The pressure in the gas cell was reduced by a diaphragm pump and the spectrum was measured soon after evacuation. The result is shown in Figure 4(b). A second, unknown absorption peak appeared at $2.585 \mathrm{THz}$. The cell was then left untouched and the spectra were measured after 30 and 60 minutes. As shown in Figures 4c \& 4d, the absorption peak at 2.585 $\mathrm{THz}$ has disappeared, while at the same time, the intensity of the peak at $2.569 \mathrm{THz}$ has increased. The peak at $2.585 \mathrm{THz}$ is thought to be from clusters of water molecules, since the reduced pressure makes the temperature lower for water molecules, so the hydrogen bonds of some water molecules become tighter, forming clusters and giving rise to the absorption peak at the higher frequency. It has been reported that there is a water trimer with an absorption line at 2.61 THz due to $k=3^{\ell} \leftarrow 0$ of $\left(\mathrm{H}_{2} \mathrm{O}\right)_{3}$ [24]. For stable water clusters, the $\left(\mathrm{H}_{2} \mathrm{O}\right)_{21} \mathrm{H}^{+}$cluster has many absorption lines in the frequency range from 1.5 to $6 \mathrm{THz}$ [25]. More experiments are needed in order to further this discussion. 


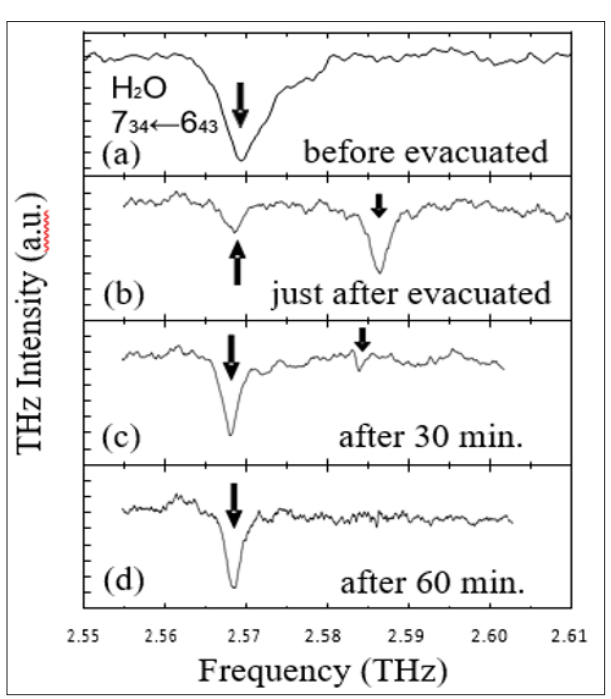

Figure 4: THz spectra of water vapor at a pressure of 14.6 $\mathrm{kPa}$ in a vapor-liquid coexistence: (a) before evacuation, (b) just after evacuation, (c) 30 min after evacuation and (d) 60 min after evacuation.

\section{Conclusion}

A LD pumped CW THz radiation generator was combined with a gas cell to obtain high resolution $\mathrm{THz}$ spectra of water vapor. In the spectra of a mixture of saturated water vapor and ethanol at room temperature, distinct spectral absorption lines for water and ethanol can be observed at around $2.46 \mathrm{THz}$. The interaction of water polar molecules is discussed based on the THz spectra of water vapor with different humidity levels at atmospheric pressure. Furthermore, THz spectra of water were obtained in a vapor-liquid coexistence at a pressure of $14.6 \mathrm{kPa}$, in which an absorption peak at $2.585 \mathrm{THz}$ appeared just after the gas cell was evacuated. This peak may be due to clusters of water molecules.

\section{Acknowledgment}

This study is partially supported by "Fundamental Research and Human Resources Development Program for Nuclear Decommissioning related to Integrity Management of Critical Structures including Primary Containment Vessel and Reactor Building, and Fuel Debris Processing and Radioactive Waste Disposal" carried out under the Center of World Intelligence Project for Nuclear S\&T and Human Resource Development by the Ministry of Education, Culture, Sports, Science and Technology of Japan.

\section{References}

1. Kawase K, Ogawa Y, Watanabe Y, Inoue H (2003) Non-destructive terahertz imaging of illicit drugs using spectral fingerprints. Opt Express 11(20): 2549-2554.

2. Nagatsuma T, Horiguchi S, Minamikata Y, Yoshimizu Y, Hisatake $S$, et al. (2013) Terahertz wireless communications based on photonicstechnologies. Opt Express 21(20): 23736-23747.

3. Takahashi S, Hamano T, Nakajima K, Tanabe T, Oyama Y (2014) Observation of damage in insulated copper cables by THz imaging. NDT \& E Int 61: 75-79.

4. Nakamura Y, Kariya H, Sato A, Tanabe T, Nishihara K, et al. (2014) Nondestructive Corrosion Diagnosis of Painted Hot-Dip Galvanizing Steel Sheets by Using THz Spectral Imaging. Corros Eng 63(9): 411-416.
5. Jackson JB, Bowen J, Walker G, Labaune J, Mourou G, et al. (2011) A Survey of Terahertz Applications in Cultural Heritage Conservation Science. IEEE Trans Terahertz Sci Tech 1(1): 220-231.

6. Matsuura S, Tani M, Abe H, Sakai K, Ozeki H, et al. (1998) High-Resolution Terahertz Spectroscopy by a Compact Radiation Source Based on Photomixing with Diode Lasers in a Photoconductive Antenna. J Mol spectrosc 187(1): 97-101.

7. Hoshina H, Seta T, Iwamoto T, Kasai Y, Hosako I, et al. (2008) Measurement of Precise Pressure Broadening Parameters of Water Vapor with a Terahertz Time Domain Spectrometer. J Quant Spectrosc Radiat Transf 109(12-13): 2303-2314.

8. Tonouchi M (2007) Cutting-edge terahertz technology. Nat Photonics 1: 97-105.

9. Schaar JE, Vodopyanov KL, Fejer MM (2007) Intracavity terahertz-wave generation in a synchronously pumped optical parametric oscillator using quasi-phase-matched GaAs. Optics Lett 32(10): 1284-1286.

10. Laman N, Harsha SS, Grischkowsky D, Melinger JS (2008) HighResolution Waveguide $\mathrm{THz}$ Spectroscopy of Biological Molecules. Biophys J 94(3): 1010-1020.

11. Kawase K, Shikata J, Ito H (2002) Terahertz wave parametric source. J Phys D Appl Phys 35: R1-R14.

12. Tanabe T, Suto K, Nishizawa J, Saito K, Kimura T (2003) Tunable terahertz wave generation in the 3- to 7-THz region from GaP. Appl Phys Lett 83(2): 237-239.

13. Vitiello MS, Tredicucci A (2011) Tunable Emission in THz Quantum Cascade Lasers. IEEE Trans. Terahertz Sci Tech 1(1): 76-84.

14. Jung S, Jiang Y, Vijayraghavan K, Jiang A, Demmerle F, et al. (2015) Recent Progress in Widely Tunable Single-Mode Room Temperature Terahertz Quantum Cascade Laser Sources. IEEE J. Sel. TOP. Quant. 21(6): 1200710.

15. Irimajiri Y, Kumagai M, Morohashi I, Kawakami A, Nagano S, et al. (2016) Precise Evaluation of a Phase-Locked THz Quantum Cascade Laser. IEEE Trans Terahertz Sci Tech 6(1): 115-120.

16. Nishizawa J, Tanabe T, Suto K, Watanabe Y, Sasaki T, et al. (2006) Continuous-Wave Frequency-Tunable Terahertz-Wave Generation from GaP. IEEE Photonics Technol Lett 18(19): 2008-2010.

17. Tanabe T, Ragam S, Oyama Y (2009) Continuous wave terahertz wave spectrometer based on diode laser pumping: Potential applications in high resolution spectroscopy. Rev Sci Instrum 80(11): 113105.

18. Benedict WS, Kaplan LD (1959) Calculation of Line Widths in H2O-N2 Collisions. J Chem Phys 30(2): 388-399.

19. Benedict WS, Kaplan LD (1964) Calculation of line widths in H2O-H2O and H2O-O2 collisions. J Quant Spectrosc Radiat Transfer 4(3): 453-469.

20. Gasster SD, Townes CH, Goorvitch D, Valero FPJ (1988) Foreign-gas collision broadening of the far-infrared spectrum of water vapor. J Opt Soc Am B5(3): 593-601.

21. Podobedov VB, Plusquellic DF, Fraser GT (2004) THz laser study of selfpressure and temperature broadening and shifts of water vapor lines for pressures up to $1.4 \mathrm{kPa}$. J Quant Spectrosc Radiat Transfer 87(3-4): 377-385.

22. Sagawa H, Mendrok J, Seta T, Hoshina H, Baron P, et al. (2009) Pressure broadening coefficients of induced by for Venus atmosphere. J Quant Spectrosc Radiat Transfer 110(18): 2027-2036.

23. http://spec.jpl.nasa.gov/ftp/pub/catalog/catform.html

24. Brown MG, Viant MR, Mc Laughlin RP, Keoshian CJ, Michael E, et al. (1999) Quantitative characterization of the water trimer torsional manifold by terahertz laser spectroscopy and theoretical analysis. II. $\left(\mathrm{H}_{2} \mathrm{O}\right)_{3}$. J Chem Phys 111: 7789-7800.

25. Johnson K, Price Gallagher M, Mamer O, Lesimple A, Fletcher C, et al. (2008) Water vapor: An extraordinary terah ertz wave source under optical excitation. Phys Lett A 372(38): 6037-6040. 
ISSN: 2574-1241

DOI: 10.26717/BJSTR.2018.10.001907

Tadao Tanabe. Biomed J Sci \& Tech Res

(C) (P) This work is licensed under Creative

Submission Link: https://biomedres.us/submit-manuscript.php

$\begin{array}{ll}\text { BIOMEDICAL } & \text { Assets of Publishing with us } \\ \text { RESEARCHES } & \text { Global archiving of articles } \\ & \text { - Immediate, unrestricted online access } \\ & \text { - Rigorous Peer Review Process } \\ \end{array}$

